\title{
A novel photoelectrochromic device with dual application based on poly(3,4-alkylenedioxythiophene) thin film and an organic dye
}

\author{
Chih-Yu Hsu ${ }^{a}$, Kun-Mu Lee ${ }^{b}$, Jen-Hsien Huang ${ }^{a}$, K.R. Justin Thomas ${ }^{c}$, Jiann T. Lin ${ }^{c}$, \\ Kuo-Chuan $\mathrm{Ho}^{\mathrm{a}, \mathrm{b}, *}$ \\ a Department of Chemical Engineering, National Taiwan University, Taipei 10617, Taiwan \\ ${ }^{\mathrm{b}}$ Institute of Polymer Science and Engineering, National Taiwan University, Taipei 10617, Taiwan \\ ${ }^{\mathrm{c}}$ Institute of Chemistry, Academia Sinica, Taipei 11529, Taiwan
}

\section{A R T I C L E I N F O}

\section{Article history:}

Received 14 August 2008

Received in revised form 4 September 2008

Accepted 10 September 2008

Available online $\mathrm{xxx}$

\section{Keywords:}

Dye-sensitized solar cell (DSSC)

Electron transfer

Organic dye

Photoelectrochromic device (PECD)

Poly(3,4-alkylenedioxythiophene)

\begin{abstract}
A B S T R A C T
A novel photoelectrochromic device (PECD) with dual application was studied using an electrochromic polymer thin film, poly(3,3-diethyl-3,4-dihydro-2H-thieno-[3,4-b][1,4]dioxepine) (PProDot-Et ${ }_{2}$ ), and an organic photosensitive dye, $\mathrm{FL}$ dye 1 , which was adsorbed on a $\mathrm{TiO}_{2}$ electrode. In the presence of the redox couple, the electron transfer reaction at the electrolyte/PProDot-Et $\mathrm{E}_{2}$ conducting polymer interface was found to determine the electrochromism of the PECD. The rate constants of the electron transfer $\left(k_{0}\right)$ for different redox couples at the PProDot-Et ${ }_{2}$ film were obtained by using electrochemical impedance spectroscopy (EIS) and were correlated with the coloration depth of the thin film. Since $\mathrm{I}^{-} / \mathrm{I}_{3}{ }^{-}$and $\mathrm{Br}^{-} / \mathrm{Br}_{3}{ }^{-}$ couples possess the highest and the lowest $k_{0}$ value, respectively, the PECD was investigated using these two redox couples under constant light illumination of $50 \mathrm{~mW} \mathrm{~cm}^{-2}$. The $\mathrm{I}^{-} / \mathrm{I}_{3}{ }^{-}$couple provided a higher short circuit current density $\left(J_{S C}\right)$ when acting as a dye-sensitized solar cell (DSSC), thus the switching response upon coloration (ca. $3 \mathrm{~s}$ ) was much faster than that of the PECD using the $\mathrm{Br}^{-} / \mathrm{Br}_{3}{ }^{-}$couple (ca. $2 \mathrm{~min}$ ). On the contrary, the PECD using $\mathrm{Br}^{-} / \mathrm{Br}_{3}{ }^{-}$exhibited a larger transmittance attenuation of $c a .33 .7 \%$, in comparison to that using $\mathrm{I}^{-} / \mathrm{I}_{3}{ }^{-}$(ca. $\left.14.1 \%\right)$.
\end{abstract}

(C) 2008 Elsevier B.V. All rights reserved.

\section{Introduction}

A photoelectrochromic device (PECD) can be designed to include an extra electrochromic layer in a dye-sensitized solar cell (DSSC). In such a design, PECD acts both as a DSSC to deliver power and a switchable electrochromic device (ECD) in one without supplying an external voltage. The photoelectrochromism was first realized in a composite film consisted of Prussian blue on titanium dioxide $\left(\mathrm{TiO}_{2}\right)$ layer [1]. Afterward, Bechinger et al. [2] changed the cell configuration to two separate electrodes, using a dyesensitized $\mathrm{TiO}_{2}$ and a cathodically coloring material $\left(\mathrm{WO}_{3}\right)$, to obtain the transmittance attenuation $(\Delta T)$ of $18 \%$. The separated type PECD offer dual application since the generated electrons can either be transported out of the device as a power source or be charged within the device as an energy saving device,

\footnotetext{
is Presented at the 2nd International Conference on Functional Materials and Devices 2008 (ICFMD-2008), Kuala Lumpur, Malaysia, 16-19 June 2008.

* Corresponding author at: Department of Chemical Engineering, National Taiwan University, Taipei 10617, Taiwan. Tel.: +88622366 0739; fax: +88622362 3040.

E-mail address: kcho@ntu.edu.tw (K.-C. Ho)
}

when compared with the original composited-film type. The most commonly investigated PECD system using Ru-dye as the photoactive species and $\mathrm{WO}_{3}$ film as the electrochromic layer. And yet, the high cost involving in the preparation of $\mathrm{WO}_{3}$ films and the limited supply of Ru-dyes with expensive rare metal are disadvantageous for the PECDs applications. Accordingly, the use of electrochromic polymer films made by a simple electrodeposition method on the separated side against the $\mathrm{TiO}_{2}$ /organic dye layer provides a low-cost possibility and a dual usage for the PECDs.

In our earlier study [3], poly(3,4-ethylenedioxythiophene) (PEDOT) was used as the electrochromic layer of a PECD for its good coloration efficiency $\left(280 \mathrm{~cm}^{2} \mathrm{C}^{-1}\right)$. PEDOT belongs to a derivative of poly(3,4-alkylenedioxythiophene)s (PXDOTs), and poly(3,3-diethyl-3,4-dihydro-2H-thieno-[3,4- $b][1,4]$ dioxepine) (PProDot-Et ${ }_{2}$ ) was reported [4] to exhibit enhanced properties among PXDOTs with composite coloration efficiency of $505 \mathrm{~cm}^{2} \mathrm{C}^{-1}$. It was reported that, in the presence of redox active species, e.g. $\mathrm{I}^{-} / \mathrm{I}_{3}{ }^{-}$redox couple in the PECD systems, the electron transfer process between the conducting polymers and the redox couples would take place [5]. This explains why a darkening process was observed in this study when the electrolyte containing 
the redox couple was brought in contact with PProDot-Et ${ }_{2}$ thin film without an external biasing.

In this work, the darkening process for the PProDot-Et ${ }_{2}$ thin films induced by the electron transfer in the presence of redox couples is analyzed by electrochemical impedance spectroscopy (EIS). With the basic understanding of the electron transfer at the PProDot-Et 2 polymer/electrolyte interface, the PECDs would be fabricated, in which the organic dye, FL dye1 ((E)-2-cyano-3-(5-(9,9-diethyl-7-(naphthalen-1yl(phenyl)amino)-9H-fluoren-2-yl)thiophen-2-yl)acrylic acid), reported previously [6] showed a good performance in DSSC [7]. To the best of our knowledge, for the first time a PECD with dual application for ECD and DSSC is integrated in one device. This design is more economical than a DSSC-ECD combined tandem cell [8].

\section{Experimental}

The monomer, 3,4-(2',2'-diethylpropylene $)$-dioxythiophene (ProDot-Et 2 ), was purchased from Aldrich and so were $\mathrm{LiClO}_{4}$, $\mathrm{KSeCN}$, tetrabutylammonium bromide ( $\mathrm{TBABr}$ ), and tetrabutylammonium chloride (TBACl). Lil and poly(ethyleneglycol) (PEG) (M.W. 20,000) were obtained from Merck. The above chemicals were used as received. Acetonitrile (ACN), 3-methoxypropionitrile (MPN) and tertiary butanol were also obtained from Merck and water molecules were removed by molecular sieves ( $4 \AA$ ).
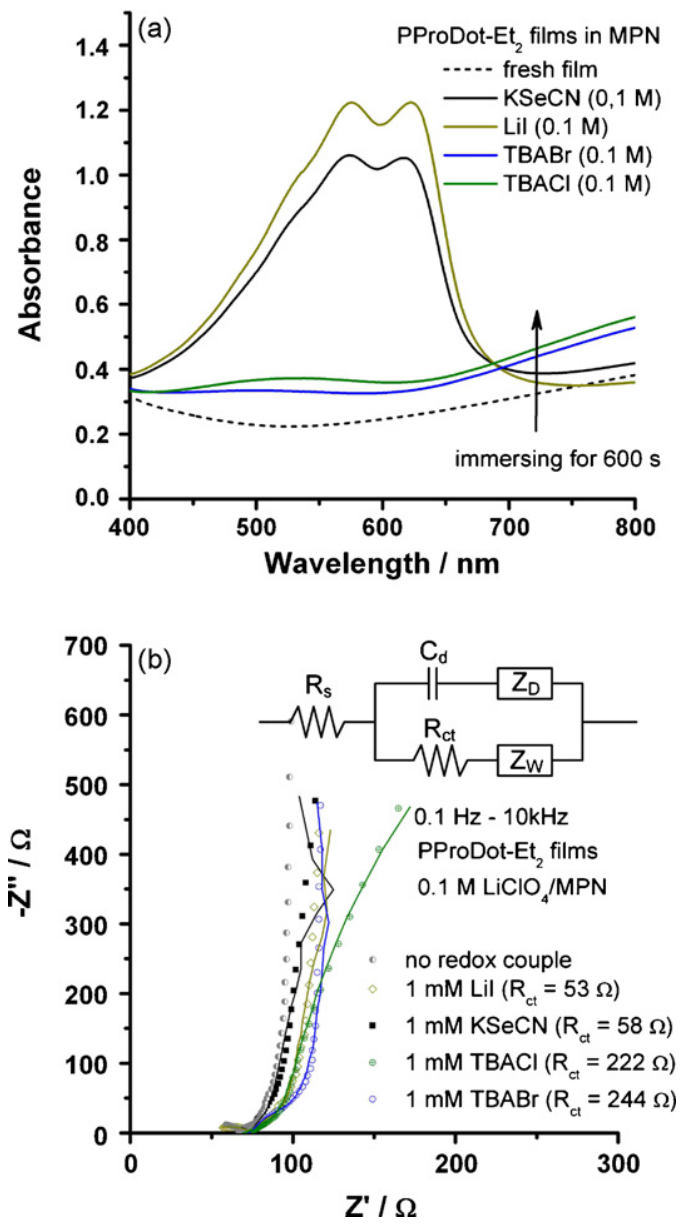

Fig. 1. (a) Absorbance spectra of PProDot-Et $t_{2}$ thin films after immersing in $0.1 \mathrm{M}$ $\mathrm{LiClO}_{4} / \mathrm{MPN}$ solution containing different redox couples $(0.1 \mathrm{M})$ for $600 \mathrm{~s}$, and (b) the corresponding Nyquist plots with the experimental data (dots) and the fitting data (lines).
The cathodically coloring PProDot-Et ${ }_{2}$ thin film was electropolymerized from the bath solution composed of $10 \mathrm{mM}$ monomer and $0.1 \mathrm{M} \mathrm{LiClO}_{4}$ in ACN using a three-electrode system with a potentiostat (Autolab Eco Chemie, PGSTAT 30). By applying a constant potential of $1.2 \mathrm{~V}$ (vs. $\left.\mathrm{Ag} / \mathrm{Ag}^{+}\right)$onto an ITO glass $\left(R_{\mathrm{sh}}=10 \Omega / \square\right)$ of $1.0 \mathrm{~cm}^{2}$ active area, the passed charge was kept at $40 \mathrm{mC} \mathrm{cm}^{-2}$. The synthetic method of the $\mathrm{TiO}_{2}$ nanoparticles was reported [9] and followed, and the electrode fabrication was carried out according to our previous method [7]. An active area of FTO glass $\left(R_{\mathrm{sh}}=25 \Omega / \square\right)$ of $1.0 \mathrm{~cm}^{2}$ was selected from the sintered electrode and then immersing in $0.3 \mathrm{mM}$ solution of FL dye 1 in ACN and tertiary butanol (in the volume ratio of $1: 1$ ) for $24 \mathrm{~h}$. The experimental procedure to synthesize FL dye 1 was described elsewhere [6].

The spectral variation of the PProDot-Et 2 films were carried out by a spectrophotometer (Shimadzu, UV 1601PC). For the EIS analysis, the PProDot-Et ${ }_{2}$ electrodes were characterized using a three-electrode system by the above-mentioned potentiostat equipped with FRA2 module in the presence of $1.0 \mathrm{mM}$ redox couples $\left(\mathrm{I}^{-} / \mathrm{I}_{3}{ }^{-} ; \mathrm{SeCN}^{-} /(\mathrm{SeCN})_{2} ; \mathrm{Cl}^{-} / \mathrm{Cl}_{3}{ }^{-} ; \mathrm{Br}^{-} / \mathrm{Br}_{3}{ }^{-}\right)$and $0.1 \mathrm{M} \mathrm{LiClO}_{4}$ in MPN. The impedance spectra were recorded at the formal potential of each redox couples in the frequency ranging from $0.1 \mathrm{~Hz}$ to $10 \mathrm{kHz}$ with sinusoidal signal (single sine) and an ac amplitude $\left(\Delta E_{\mathrm{ac}}\right)$ of $10 \mathrm{mV}$. The impedance spectra were fitted to an equivalent circuit model proposed by Sundfors et al. [10], and the model parameters were obtained by ZView software.

The PECD was sandwiched using the electrochromic and photoactive electrodes separated by an electrolyte of $0.1 \mathrm{M}$ redox couples and $0.1 \mathrm{M} \mathrm{LiClO}_{4}$ in MPN. The optical performance and the photoelectrochemical charateristics of the PECD under constant light illumination of $50 \mathrm{~mW} \mathrm{~cm}{ }^{-2}$ were measured by a portable spectrometer (Ocean Optronics, USB 2000) and the above mentioned potentiostat, respectively. The experimental setup is in common with our previous system [3].

\section{Results and discussion}

\subsection{Electron transfer kinetics between redox couples and PProDot-Et 2 thin films}

The electron transfer reaction between conducting polymers and redox couples had been studied on PEDOT or polypyrrole and its derivative [10-12]. The results indicated that the electrons could transfer across the polymer-electrolyte solution interface and reach the polymer film so as to alter the redox state of the conducting polymers. Therefore, if the conducting polymer possesses electrochromic property, the color change of the polymer thin film can be observed. Fig. 1(a) shows the absorbance spectra for PProDot-Et ${ }_{2}$ thin films, which were brought in contact with different redox couples for $600 \mathrm{~s}$ upon receiving electrons from the redox couples. As can be seen in Fig. 1(a), the depth of the darkening process depends on the redox couple, with the $\mathrm{I}^{-} / \mathrm{I}_{3}{ }^{-}$and $\mathrm{Br}^{-} / \mathrm{Br}_{3}{ }^{-}$ redox couples gave the most and least transmittance attenuation, respectively. Since different redox couples result in different transmittance attenuation in the PProDot-Et ${ }_{2}$ film, it is expected to affect that of the PECD.

The change in transmittance depends on the facility of the electron transfer from the redox couples to the polymer films. Fig. 1(b) shows the impedance spectra of PProDot-Et ${ }_{2}$ thin films characterized under various redox couples of $1.0 \mathrm{mM}$ and $0.1 \mathrm{M} \mathrm{LiClO}_{4}$ as the supporting electrolyte. The presence of redox couples affects the impedance characteristic when comparing with that without using redox couple, which is a typical behavior observed under similar condition [10]. The rate constant of the electron transfer $\left(k_{0}\right)$ can 
be obtained by the following equation [10]:

$k_{0}=\frac{R T}{n^{2} F^{2} R_{\mathrm{ct}} A C}$

where $A$ is the area of the electrode, $C$ is the bulk concentration of the redox couple and $R_{\mathrm{ct}}$ is the charge transfer resistance obtained by fitting the EIS data to the equivalent circuit as shown in the inset of Fig. 1(b). The other symbols have their usual electrochemical meanings.

The fitting results can be seen in Fig. 1(b), where the solid lines represent the modeling results obtained by fitting the dotted experimental data and the fitted $R_{\mathrm{ct}}$ values were also listed behind each legend. The values of $k_{0}$ were consequently calculated through Eq. (1) as following: $1.3 \times 10^{-3} \mathrm{~cm} \mathrm{~s}^{-1}$ for $\mathrm{I}^{-} / \mathrm{I}_{3}{ }^{-}$, $1.2 \times 10^{-3} \mathrm{~cm} \mathrm{~s}^{-1}$ for $\mathrm{SeCN}^{-} /(\mathrm{SeCN})_{2}, 3.0 \times 10^{-4} \mathrm{~cm} \mathrm{~s}^{-1}$ for $\mathrm{Cl}^{-} / \mathrm{Cl}_{3}{ }^{-}$, and $2.8 \times 10^{-4} \mathrm{~cm} \mathrm{~s}^{-1}$ for $\mathrm{Br}^{-} / \mathrm{Br}_{3}{ }^{-}$. It is obvious that the value of $k_{0}$ is related to the absorbance increment (Fig. 1(a)) for each redox couple that was brought in contact with the PProDot-Et ${ }_{2}$ thin film. The relationship between the absorbance change and the key parameters in the electron transfer process can be established by considering the definition of the coloration efficiency $(\eta)$ for an electrochromic material and the exchange current density $\left(j_{0}\right)$ expression [13] as follows:

$\eta=\frac{\Delta A}{q}$

$j_{0}=n F k_{0} C \exp \left[\frac{-\alpha n F\left(E_{\mathrm{eq}}-E^{0^{\prime}}\right)}{R T}\right]$

where $\Delta A$ is the absorbance change, $q$ is the inserted charge density, $\alpha$ is the transfer coefficient, $E_{\text {eq }}$ is the equilibrium potential of the electrode and $E^{0^{\prime}}$ is the formal potential of the redox couples in the electrolytic solution. Under our experimental condition, i.e. $E_{\mathrm{eq}}=E^{0^{\prime}}$, Eqs. (2) and (3) can be combined as:

$\Delta A=n F k_{0} C t \eta$

where $t$ is the reaction time and we assumed that $q$ is contributed steadily from $j_{0}$. Namely, $q=j_{0} t$. Therefore, $\Delta A$ is proportional to $k_{0}$. However, the reaction time before reaching the equilibrium for the redox couples and the PProDot-Et ${ }_{2}$ thin film is unknown and the coloration efficiency also depends on the inserted charge density of the film, which is the characteristic of the conducting polymer materials [14]. Even so, it is still useful to know according to Eq. (4), that the value of $k_{0}$ is related to the absorption change. In addition, we also recorded the change in the open circuit potential of PProDot-Et ${ }_{2}$ electrodes, before and after the addition of redox couples (not shown), and the decrement in the open circuit potential means that the electron-receiving process is also proportional to the value of $k_{0}$. It is shown that the electrons transfer to the cathodically coloring PProDot-Et $t_{2}$ thin film depends kinetically on the value of $k_{0}$, which depends on the redox couple employed, and finally reaches static equilibrium.

\subsection{Performances of the PECDs}

According to the kinetic consideration, the redox couple of $\mathrm{Br}^{-} / \mathrm{Br}_{3}{ }^{-}$was selected for improving the bleached state performance of the PECD to compete with the PECD containing the $\mathrm{I}^{-} / \mathrm{I}_{3}{ }^{-}$couple. The photocurrent-voltage $(I-V)$ characteristic and optical performance of the PECDs composed of PProDot-Et 2 electrochromic thin film and $\mathrm{FL}$ dye $1-\mathrm{TiO}_{2}$ photoactive layer under the light illumination of $50 \mathrm{~mW} \mathrm{~cm}^{-2}$ are shown in Figs. 2 and 3, respectively. According to the inset of Fig. 3, the characteristic wavelength of the PECD was determined to be $c a .620 \mathrm{~nm}$. Table 1 summarizes

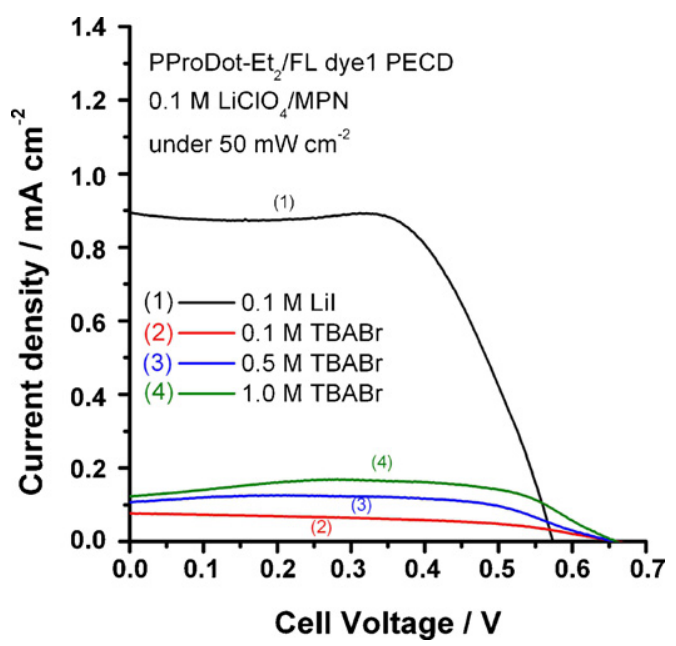

Fig. 2. Photocurrent-voltage characteristic of PProDot-Et $t_{2} / \mathrm{FL}$ dye $1-\mathrm{TiO}_{2} \mathrm{PECDs}$ under $50 \mathrm{~mW} \mathrm{~cm}^{-2}$ illumination with various conditions of the redox couples.

the optical and photoelectrochemical performance obtained from Figs. 2 and 3.

As a result of a higher value of $k_{0}$ for $\mathrm{I}^{-} / \mathrm{I}_{3}{ }^{-}$, the facility of electron transfer leads to faster regeneration of the FL dye 1 on the $\mathrm{TiO}_{2}$ layer and thus the $J_{S C}$ output from the device is much higher than that using $\mathrm{Br}^{-} / \mathrm{Br}_{3}{ }^{-}$with a lower value of $k_{0}$, as shown in Table 1. When the electron is transported to the PProDot-Et $t_{2}$ film, higher the current, faster the electrochromic reaction, and a response time of $c a .3 \mathrm{~s}$ supports the advantageous of using $\mathrm{I}^{-} / \mathrm{I}_{3}{ }^{-}$redox couple. Besides, it is worth to note that the $J_{\mathrm{Sc}}$ value of only $0.89 \mathrm{~mA} \mathrm{~cm}^{-2}$ can provide faster response for the electrochromic reaction and the fill factor (FF) of this PECD is also high (FF 0.64), as shown in Fig. 2. However, in the presence of $0.1 \mathrm{M} \mathrm{LiI}$, the bleached state transmittance of the PECD limits the transmittance attenuation with only ca. $14.1 \%$, as compared with $c a$. $25.9 \%$ when using $0.1 \mathrm{M}$ TBABr. Subsequently, the concentration of $\mathrm{TBABr}$ was increased to improve the response time by increasing the $J_{S C}$ for faster regeneration of the dye. Indeed, the response time became shorter when the concentration was increased from 0.1 to $1.0 \mathrm{M}$ as shown in Fig. 3. In fact, the response time of less than 2 min was reached, which is faster than other PECDs reported [2,3,15]. Moreover, the bleached state trans-

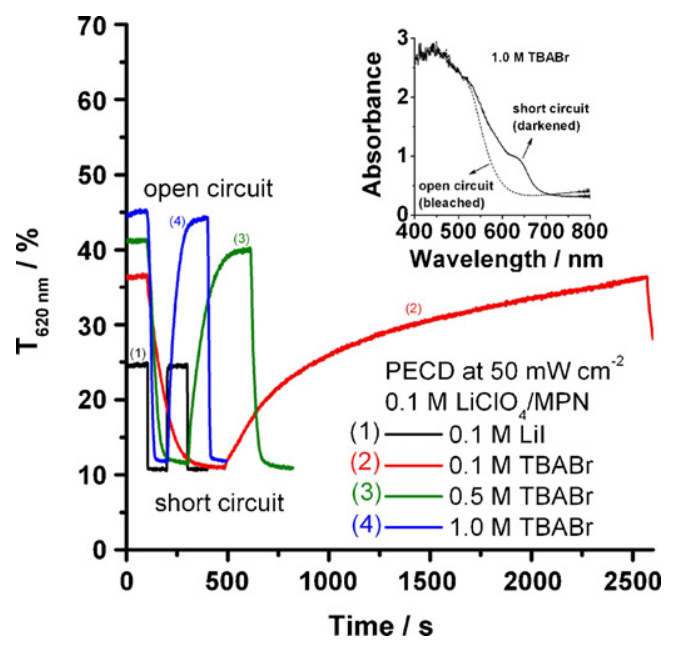

Fig. 3. Transmittance responses at $620 \mathrm{~nm}$ of $\mathrm{PProDot}_{-\mathrm{Et}} / \mathrm{FL}$ dye $1-\mathrm{TiO}_{2} \mathrm{PECDs}$ under $50 \mathrm{~mW} \mathrm{~cm}^{-2}$ illumination with various conditions of the redox couples. The inset shows the absorbance spectra for $1.0 \mathrm{M} \mathrm{TBABr}$ case under the open circuit (bleaching) and the short circuit (darkening) conditions. 
Table 1

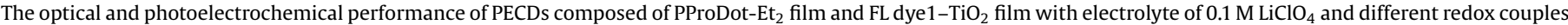
in MPN.

\begin{tabular}{|c|c|c|c|c|c|c|c|c|c|}
\hline \multirow[t]{2}{*}{ Redox couples in the PECD } & \multicolumn{5}{|c|}{ Acting as an ECD } & \multicolumn{4}{|c|}{ Acting as a DSSC } \\
\hline & $T_{\mathrm{b}}{ }^{\mathrm{a}}(\%)$ & $T_{\mathrm{d}}{ }^{\mathrm{a}}(\%)$ & $\Delta T^{\mathrm{a}}(\%)$ & $\tau_{\mathrm{b}}{ }^{\mathrm{b}}(\mathrm{s})$ & $\tau_{\mathrm{d}}{ }^{\mathrm{b}}(\mathrm{s})$ & $J_{S C}\left(\mathrm{mAcm}^{-2}\right)$ & $V_{\mathrm{OC}}(\mathrm{V})$ & $\mathrm{FF}$ & $\operatorname{PCE}^{\mathrm{c}}(\%)$ \\
\hline $0.1 \mathrm{M}$ Lil & 24.7 & 10.6 & 14.1 & 3 & 3 & 0.89 & 0.57 & 0.64 & 0.65 \\
\hline $0.1 \mathrm{M} \mathrm{TBABr}$ & 36.6 & 10.7 & 25.9 & 1823 & 207 & 0.08 & 0.66 & 0.47 & 0.05 \\
\hline $0.5 \mathrm{M}$ TBABr & 41.3 & 11.4 & 29.9 & 166 & 87 & 0.11 & 0.66 & 0.69 & 0.10 \\
\hline 1.0 M TBABr & 45.3 & 11.6 & 33.7 & 96 & 36 & 0.12 & 0.66 & 0.90 & 0.14 \\
\hline
\end{tabular}

a The values of the transmittance are obtained from data in Fig. 3.

b The response time is calculated from the transmittance change when it reaches $95 \%$ of the final value.

c The power conversion efficiency (PCE).

mittance at higher concentration of $\mathrm{Br}^{-} / \mathrm{Br}_{3}{ }^{-}$was also increased and the transmittance attenuation at $620 \mathrm{~nm}$ achieved $c a .33 .7 \%$. The improvement in the bleached state transmittance at higher concentration of $\mathrm{Br}^{-} / \mathrm{Br}_{3}{ }^{-}$is attributed to more ions insertion into the PProDot-Et $t_{2}$ thin film upon the anodic bleaching process. However, the $I-V$ characteristic of the PECDs at higher $\mathrm{Br}^{-} / \mathrm{Br}_{3}{ }^{-}$ concentration bulge out, which may result from the stronger redox property of PProDot-Et 2 . To sum up, this novel PECD offers a transmittance attenuation of $33.7 \%$ as the ECD or $V_{\mathrm{OC}}$ of $0.57 \mathrm{~V}$ and $J_{\mathrm{SC}}$ of $0.89 \mathrm{~mA} \mathrm{~cm}^{-2}$ as the DSSC for dual application in one device. It provides a new strategy in redesigning a new class of PECD $[8,15]$.

\section{Conclusions}

This study presents a novel photoelectrochromic device using the PProDot-Et ${ }_{2}$ electrochromic film and the FL dye1- $\mathrm{TiO}_{2}$ photoactive layer for dual application as a photoanode/photocathode combination. The bleached state transmittance of the device was found to be dependent on the interaction between the redox couples and the PProDot-Et 2 polymer. An analysis of the electron transfer, based on the EIS, revealed that the absorption change of the PProDot-Et $t_{2}$ thin film is proportional to the rate constant of the electron transfer $\left(k_{0}\right)$ of the redox couples. The $\mathrm{I}^{-} / \mathrm{I}_{3}{ }^{-}$couple with high $k_{0}$ value facilitates the coloration of the PProDot-Et $t_{2}$ thin film and results in a low-bleached state transmittance of the PECD, thus giving the transmittance attenuation value of only ca. $14.1 \%$. Nevertheless, this configuration provides a very fast switching response of $c a .3 \mathrm{~s}$ when acting as an ECD. The same configuration gives a very high-photocurrent density with $J_{\mathrm{SC}}$ of $0.89 \mathrm{mAcm}^{-2}$ and $V_{\mathrm{OC}}$ of $0.57 \mathrm{~V}$ when acting as a DSSC. On the other hand, the PECD using $\mathrm{Br}^{-} / \mathrm{Br}_{3}-$ with a lower $k_{0}$ value provides larger transmittance change of $\mathrm{ca} .33 .7 \%$ at high $\mathrm{Br}^{-} / \mathrm{Br}_{3}{ }^{-}$concentration, while the response time is slower (less than $2 \mathrm{~min}$ ). As expected, this
PECD only deliver a $J_{S C}$ value of $0.12 \mathrm{mAcm}^{-2}$ when acting as a DSSC. Finally, it is necessary to individually optimize the thicknesses of both photoanode (photoactive layer) and photocathode (electrochromic layer) for each application in future work.

\section{Acknowledgements}

The financial support, provided by the National Taiwan University and the National Science Council of Taiwan, under grant number NSC 95-2221-E-002-353-MY2, are gratefully acknowledged.

\section{References}

[1] D.W. DeBerry, A. Viehbeck, J. Electrochem. Soc. 130 (1983) 249-251.

[2] C. Bechinger, S. Ferrere, A. Zaban, J. Sprague, B.A. Gregg, Nature 383 (1996) 608-610.

[3] J.Y. Liao, K.C. Ho, J. New Mater. Electrochem. Syst. 8 (2005) 37-47.

[4] C.L. Gaupp, D.M. Welsh, J.R. Reynolds, Macromol. Rapid Commun. 23 (2002) $885-889$.

[5] R.A. Bull, F.R.F. Fan, A.J. Bard, J. Electrochem. Soc. 129 (1982) 1009-1015.

[6] K.R. Justin Thomas, J.T. Lin, Y.C. Hsu, K.C. Ho, Chem. Commun. (2005) 40984100.

[7] K.M. Lee, V. Suryanarayanan, K.C. Ho, K.R. Justin Thomas, J.T. Lin, Sol. Energy Mater. Sol. Cells 91 (2007) 1426-1431.

[8] K.S. Ahn, S.J. Yoo, M.S. Kang, J.W. Lee, Y.E. Sung, J. Power Sources 168 (2007) 533-536.

[9] M. Grätzel, J. Sol-Gel Sci. Technol. 22 (2001) 7-13.

[10] F. Sundfors, J. Bobacka, A. Ivaska, A. Lewenstam, Electrochim. Acta 47 (2002) 2245-2251.

[11] K. Maksymiuk, K. Doblhofer, Electrochim. Acta 39 (1994) 217-227.

[12] J. Bobacka, Z. Gao, A. Ivaska, J. Electroanal. Chem. 368 (1994) 33-41.

[13] A.J. Bard, L.R. Faulkner, Electrochemical Methods: Fundamentals and Applications, Second ed., Wiley, New York, 2001, p. 98.

[14] C.L. Gaupp, D.M. Welsh, R.D. Rauh, J.R. Reynolds, Chem. Mater. 14 (2002) 3963-3970.

[15] A. Georg, A. Georg, U.O. Krašovec, Thin Solid Films 502 (2006) 246-251. 